\title{
Pre-Town Meeting on Spin Physics at an Electron-Ion Collider ${ }^{a}$
}

Elke-Caroline Aschenauer, ${ }^{1}$ Ian Balitsky, ${ }^{2,3}$ Leslie Bland, ${ }^{1}$ Stanley J. Brodsky, ${ }^{4}$ Matthias Burkardt,${ }^{5}$ Volker Burkert, ${ }^{3}$ Jian-Ping Chen, ${ }^{3}$ Abhay Deshpande, ${ }^{6,7}$ Markus Diehl, ${ }^{8}$ Leonard Gamberg, ${ }^{9}$, † Matthias Grosse

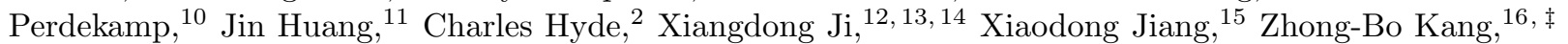
Valery Kubarovsky, ${ }^{3}$ John Lajoie, ${ }^{17}$ Keh-Fei Liu ${ }^{18}$ Ming Liu, ${ }^{15}$ Simonetta Liuti, ${ }^{19}$ Wally Melnitchouk, ${ }^{3}$ Piet Mulders, ${ }^{20}$ Alexei Prokudin,, 3 , 3 Andrey Tarasov, ${ }^{3}$ Jian-Wei Qiu, ${ }^{21,}{ }^{22}$ Anatoly Radyushkin,,${ }^{2,3}$ David Richards, ${ }^{3}$ Ernst Sichtermann, ${ }^{23}$ Marco Stratmann, ${ }^{24}$ Werner Vogelsang, ${ }^{24}$ and Feng Yuan ${ }^{23}$

${ }^{1}$ Physics Department, Brookhaven National Laboratory, Upton, New York 11973, USA

${ }^{2}$ Physics Department, Old Dominion University, Norfolk, Virginia 23529, USA

${ }^{3}$ Jefferson Lab, 12000 Jefferson Avenue, Newport News, Virginia 23606, USA

${ }^{4}$ SLAC National Accelerator Laboratory, Stanford University, Stanford, California94309, USA

${ }^{5}$ Department of Physics, New Mexico State University, Las Cruces, NM 88003-8001, USA

${ }^{6}$ RIKEN BNL Research Center, Brookhaven National Laboratory, Upton, New York 11973-5000, USA

${ }^{7}$ Department of Physics and Astronomy, Stony Brook University, SUNY, Stony Brook, New York 11794-3800, USA

${ }^{8}$ Deutsches Elektronen-Synchroton DESY, 22607 Hamburg, Germany

${ }^{9}$ Division of Science, Penn State University-Berks, Reading, PA 19610, USA

${ }^{10}$ University of Illinois at Urbana-Champaign, Urbana, Illinois 61801, USA

${ }^{11}$ Massachusetts Institute of Technology, Cambridge, Massachusetts 02139

${ }^{12}$ INPAC, Department of Physics, and Shanghai Key Lab for Particle Physics and Cosmology, Shanghai Jiao Tong University, Shanghai, 200240, P. R. China

${ }^{13}$ Center for High-Energy Physics, Peking University, Beijing, 100080, P. R. China

${ }^{14}$ Maryland Center for Fundamental Physics, University of Maryland, College Park, Maryland 20742, USA

${ }^{15}$ Los Alamos National Laboratory, Los Alamos, New Mexico 87545, USA

${ }^{16}$ Theoretical Division, Los Alamos National Laboratory, Los Alamos, New Mexico 87545, USA

${ }^{17}$ Iowa State University, Ames, Iowa 50011, USA

${ }^{18}$ Dept. of Physics and Astronomy Center for Computational

Sciences University of Kentucky, Lexington, Kentucky 40506, USA

${ }^{19}$ Department of Physics, University of Virginia, Charlottesville, Virginia 22904, USA

${ }^{20}$ Nikhef and Department of Physics and Astronomy, VU University Amsterdam, De Boelelaan 1081, NL-1081 HV Amsterdam, the Netherlands

${ }^{21}$ Physics Department, Brookhaven National Laboratory, Upton, NY 11973, USA

${ }^{22}$ C.N. Yang Institute for Theoretical Physics and Department of Physics and Astronomy, Stony Brook University, Stony Brook, NY 11794-3840, USA

${ }^{23}$ Nuclear Science Division, Lawrence Berkeley National Laboratory, Berkeley, California 94720, USA

${ }^{24}$ Institute for Theoretical Physics, Tübingen University, Auf der Morgenstelle 14, 72076 Tübingen, Germany

(Dated: November 3, 2014)

A polarized $e p / e A$ collider (Electron-Ion Collider, or EIC), with polarized proton and lightion beams and unpolarized heavy-ion beams with a variable center-of-mass energy $\sqrt{s} \sim 20$ to $\sim 100 \mathrm{GeV}$ (upgradable to $\sim 150 \mathrm{GeV}$ ) and a luminosity up to $\sim 10^{34} \mathrm{~cm}^{-2} \mathrm{~s}^{-1}$, would be uniquely suited to address several outstanding questions of Quantum Chromodynamics, and thereby lead to new qualitative and quantitative information on the microscopic structure of hadrons and nuclei. During this meeting at Jefferson Lab we addressed recent theoretical and experimental developments in the spin and the three-dimensional structure of the nucleon (sea quark and gluon spatial distributions, orbital motion, polarization, and their correlations). This mini-review contains a short update on progress in these areas since the EIC White paper [1].

a Mini-review summarizing the Informal Pre-Town Meeting at Jefferson Lab, http://www.jlab.org/conferences/pretownjlab2014/

$\dagger \operatorname{lpg} 10 @$ psu.edu

$\ddagger$ zkang@lanl.gov

$\S$ prokudin@jlab.org 


\section{Introduction:}

A group of 44 physicists from the spin physics community gathered at Jefferson Lab from August 12-15, 2014 to discuss the current status of an Electron-Ion Collider (EIC) and its impact on the future development of nuclear physics, and in particular on spin physics and the three-dimensional (" $3-\mathrm{D}$ ") structure of hadrons (with a focus on nucleon structure).

Participants were asked to prepare short presentations concerning the important scientific questions in spin physics and in particular in "3-D" nucleon structure which could be addressed and answered by an EIC. In order to facilitate discussions, the organizers appointed "advocates" and "convener-opponents" for each topic discussed. The "advocates" were given time to present their view of future impact of EIC physics, while "convener-opponents" were asked to lead and direct discussions, posing in depth questions about the content and justifications presented by "advocates". The discussions were followed by short presentations with an opportunity for ample contributions from all participants. In particular the discussions were intended to summarize developments in both theory and experiment in the last two years that followed the completion of the EIC White Paper [1].

This document summarizes the progress discussed during the "Informal Pre-town Meeting at Jefferson Lab", August 12-15, 2014. All presented talks can be found on the workshop website:

http://www.jlab.org/conferences/pretownjlab2014/program.html.

\section{General discussion:}

As a basic building block of more than $99 \%$ of the mass of the visible world, the nucleon is a confined system of strongly interacting quarks, anti-quarks, and gluons (partons). Understanding this complex structure and the nature of the strong force is a great scientific challenge and one of the paramount problems of modern nuclear physics, as described in the 2007 NSAC Long-Range Plan [2. It is an essential step in describing nuclear structure and reactions from first principles, with numerous applications to science and technology. The past century has been marked by a truly remarkable achievement: Quantum Chromodynamics (QCD), the fundamental theory of the strong force, has been tested and established. Experiment has yielded precision insights on the proton's spatial extent, on the one hand, and its momentum structure on the other, revealed the existence of a large number of soft gluons, and resulted in many surprises with nuclei and spin. The ongoing and planned programs at Jefferson Lab, RHIC and other facilities in Europe and Japan will allow us to significantly extend these studies and for the first time gain precision knowledge of the combined spatial-momentum structure of the nucleon in the region dominated by valence quarks, as well as expanding our understanding of the partonic sea of nucleons and nuclei.

A polarized $e p / e A$ Electron-Ion Collider with polarized proton and light-ion beams and unpolarized heavy-ion beams, with a variable ep center-of-mass $(\mathrm{CM}$ ) energy in the range $\sqrt{s} \sim 20$ to $\sim 100 \mathrm{GeV}$ (upgradable to $\sim 150$ $\mathrm{GeV}$ ), will be a uniquely powerful "femtoscope" to improve our understanding of the nucleon and the nucleus in the "sea" quark and gluon-dominated regime at high $Q^{2}$. With its unique versatility and state-of-the-art luminosity of up to $\sim 10^{34} \mathrm{~cm}^{-2} \mathrm{~s}^{-1}$, its high polarization, and detector capabilities (as specified in the EIC White Paper [1] and Refs. [3 5]), it will achieve a precision far beyond that of any other existing and planned facilities in the world.

The inability to observe quarks and gluons in isolation, owing to QCD confinement, presents a great challenge in studying the interior structure of nucleons and nuclei. An EIC with its unprecedented precision may provide data which help to address this challenge by probing nucleon structure with increasing accuracy. The combination of precision data from a broad range of reactions at an EIC, with commensurate advances in the theory of QCD dynamics of quarks, anti-quarks, and gluons, will lead to the discovery of new phenomena and will reveal new structures inside hadrons. In turn this will yield unprecedented understanding of nucleon and nuclear properties in terms of these elementary constituents and their dynamics, as well as the mechanism for the emergence of hadrons. Theoretical methods to apply QCD to hadronic and nuclear systems have made dramatic advances in the last two decades, but rely crucially on new experimental information for further progress.

An example is the gluon spin: An EIC can delineate with unprecedented precision, over an extended kinematic region in Bjorken-x, the full helicity structure of the proton in terms of gluons, quarks, and anti-quarks and their flavor. This will address one very important aspect of the nucleon spin puzzle.

Another example is the combined spatial and momentum parton structure in the nucleon in terms of densities, which are encoded in the transverse-momentum-dependent distributions (TMDs) and generalized parton distributions (GPDs). In addition to providing a tomographic description of the nucleon, the precision mapping of TMDs and GPDs will provide new qualitative and quantitative information about the orbital motion of the partons and, with further development of phenomenological models, and ab initio lattice calculations, quantitative information about orbital angular momentum. 


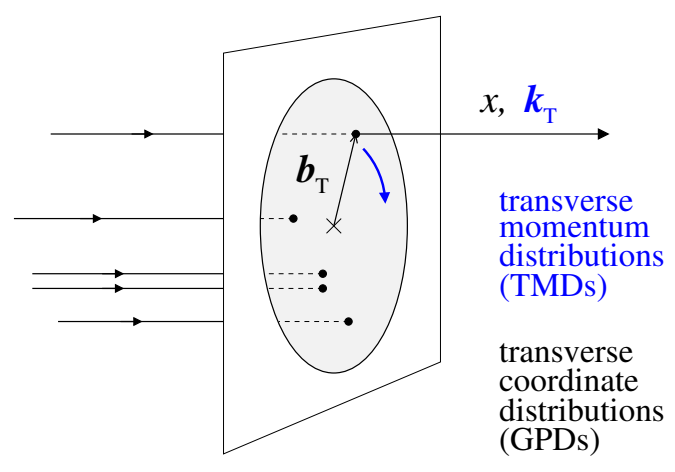

FIG. 1. Three-dimensional structure of a fast-moving nucleon. The distribution of partons (quarks, gluons) is characterized by the longitudinal momentum fraction $x$ and the transverse spatial coordinate $\boldsymbol{b}_{T}$ through the impact parameter GPDs 6 . In addition, the partons are distributed over transverse momenta $\boldsymbol{k}_{T}$, reflecting their orbital motion and interactions in the system (TMDs). Polarization distorts both the spatial and momentum distributions. Note that $\boldsymbol{b}_{T}$ and $\boldsymbol{k}_{T}$ are not Fourier conjugate; a joint description in both variables can be formulated in terms of a Wigner phase space density [7. Observables sensitive to either $\boldsymbol{b}_{T}$ or $\boldsymbol{k}_{T}$ help to establish a three-dimensional dynamical picture of the nucleon in QCD. Figure from Ref. [5].

\section{Three-dimensional structure of the nucleon in QCD:}

The nucleon in QCD represents a dynamical system of fascinating complexity. Viewed in high-energy interactions the nucleon's color field can be represented by elementary quanta with point-particle characteristics (partons), and the nucleon becomes a many-body system of quarks, anti-quarks and gluons. In contrast to non-relativistic systems, in QCD the number of constituents is not fixed, as they constantly undergo creation/annihilation processes mediated by QCD interactions. This reflects the relativistic nature of the dynamics. A high-energy scattering process takes a "snapshot" of this fast-moving system with a spatial resolution given by the inverse momentum transfer $1 / Q$. In addition to the valence quarks, the nucleon contains a "sea" of quark-anti-quark pairs. The spin and flavor quantum numbers carried by the sea, both light and heavy, are poorly constrained by present data. An EIC would be the only polarized "femtoscope" facility in the world that is capable of accessing distances $<10^{-15} \mathrm{~cm}$ inside of the polarized nucleon in the regime where sea quarks and gluons dominate.

Experimental measurements of semi-inclusive deep inelastic scattering (SIDIS) and exclusive processes such as deeply virtual Compton scattering (DVCS) lead to an understanding of a "three-dimensional" representation of the nucleon in coordinate and momentum space. New distributions related to those representations are so-called Generalized Parton Distributions and Transverse Momentum Dependent distributions, see Fig. 1. A joint description in both momentum and coordinate space can be formulated in terms of Wigner phase space densities [7, however, it remains a challenge to find a way of accessing these distributions in high-energy scattering experiments. Measurements with polarized beams of light ions and electrons are key requirements for understanding GPDs and TMDs. An EIC will measure TMDs of sea quarks through semi-inclusive measurements, in which the charge and flavor of the struck quark/anti-quark are "tagged" by detecting hadrons $\left(\pi^{ \pm}, K^{ \pm}, p, \bar{p}, \ldots\right)$ produced from its fragmentation.

Since the publication of the EIC White Paper [1, theoretical tools for QCD evolution of TMDs have been developed and have been successfully implemented in phenomenological calculations (see contributions in [8]). This progress allows one to cover much larger energy momentum ranges, and thereby implement QCD-based fits that are able to describe data coming from low to medium energies, such as Jefferson Lab 6, HERMES, COMPASS up to high energies of LHC. Recent progress in SIDIS experiments, and publication of unpolarized multiplicities from Jefferson Lab, HERMES, and COMPASS allowed for the first time successful implementation of QCD evolution of TMDs. Future measurements of the Drell-Yan process at COMPASS, FERMILAB, and RHIC will probe for the first time the universality properties of those distributions 9 12. In its report to NSAC by the subcommittee on performance measures this was identified as one of the high priority milestones (HP-13) 2]: Namely, to "Test unique QCD predictions for relations between single-transverse spin phenomena in $p p$ scattering and those observed in deep-inelastic lepton scattering". However, a truly quantitative measurement of TMDs will require the large multi-dimensional phase space and the high luminosity of an EIC, and only at this facility will we be able to explore the region of sea quarks. EIC kinematics is ideally suited to probe the interplay between non perturbative and perturbative dynamics in generating transverse momentum and to quantify the non perturbative contribution to the scale evolution of TMDs. The data from an EIC will be crucial for future progress in the development of the theory and phenomenology of TMDs as it relates to the " 3 -D" momentum structure of the nucleon and nuclei.

GPDs define the basic size and shape of the nucleon in QCD, generalizing the one-dimensional picture conveyed from the longitudinal momentum densities into a "two spatial plus one momentum"-dimensional image of the fast moving nucleon [6. Information on the transverse distribution of quarks and gluons is obtained from exclusive scattering $\gamma^{*} N \rightarrow M+N(M=$ meson, $\gamma$, heavy quarkonium). GPDs combine the concept of the quark/gluon momentum density with that of elastic nucleon form factors. Measurements of $J / \psi$ photo/electro-production with an EIC provide a unique opportunity to map the transverse spatial distribution of gluons in the nucleon above $x \sim$ few $\times 10^{-3}$ in unprecedented detail.

The EIC will map the spatial distributions of quarks and gluons over a wide range of the longitudinal momentum 


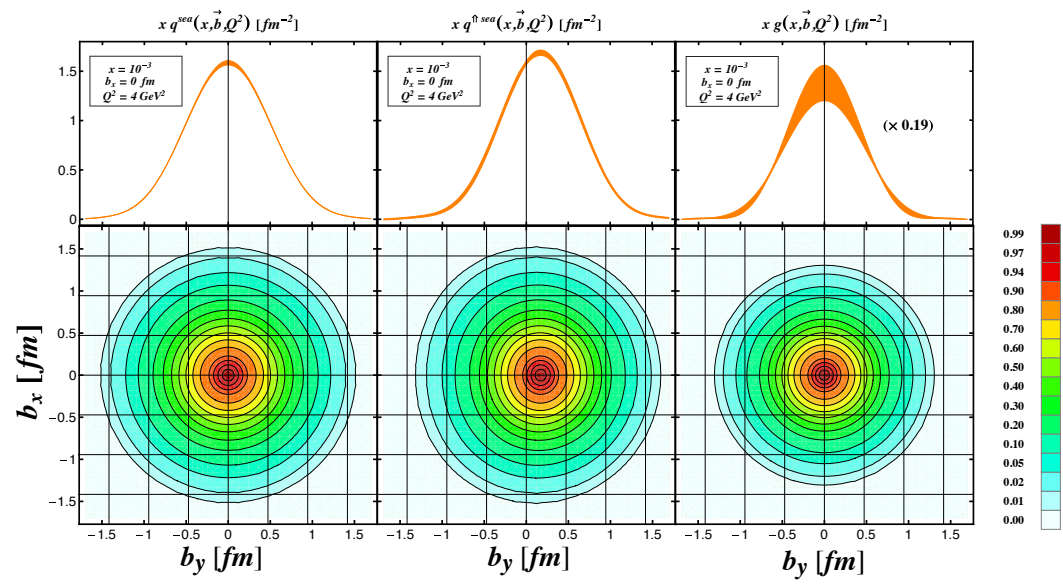

(a)

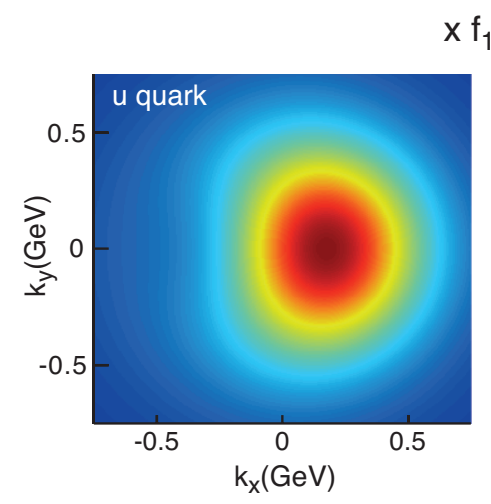

$x f_{1}\left(x, k_{T}, S_{T}\right)$

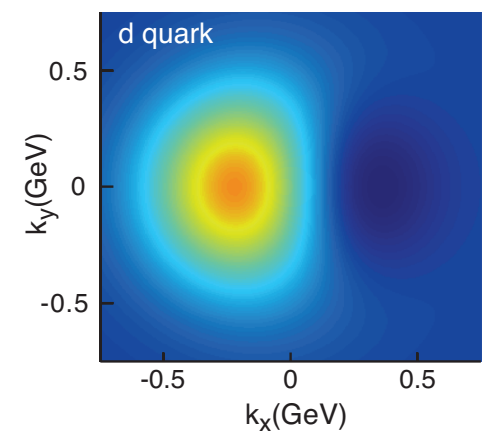

(b)

FIG. 2. Examples of three-dimensional structure: (a) Parton densities at $x=0.001$ and $Q^{2}=4 \mathrm{GeV}^{2}$ versus impact parameter $b$ obtained from a combined least-squares fit to HERA collider and EIC pseudo-data: relative densities (lower row) and their values at $b_{x}=0$ for the unpolarized sea quark parton densities of a unpolarized proton (left), a transversely polarized proton (middle), and the unpolarized gluon parton density of a unpolarized proton (right), its value is re-scaled by a factor 0.19. Figure from Ref. 13. (b) current knowledge of TMD distributions for $u$ and $d$ quarks at $x=0.1$ as function of $k_{x}$ and $k_{y}$ presented as "tomographic" slice. Figure from Ref. [1.

fraction. We expect large differences in these spatial distributions for the charge, quark-matter, and gluon-matter distributions in the region of $x<0.1$.

In the valence region $(x>0.1)$ the EIC will add a large lever arm in $Q^{2}$ to complement the data obtained from the Jefferson Lab and COMPASS programs. The up-, down-, and strange-quark distributions do not follow a simple scaling based on their current quark masses, and there is evidence from PDF fits to the HERA DIS data that there is significant strength in the gluon distributions at high $x$. In addition to the sea, generated by gluon-splitting, QCD predicts from first principles the existence of heavy quarks at large $x$ in the proton wave function. Such distributions are crucial for understanding the production of hadrons containing heavy quarks both at threshold and at high longitudinal momentum. Direct evidence for such "intrinsic" heavy quarks has been difficult to obtain from existing data [14, and an EIC has the potential to discover this component, if it exists at non-negligible levels.

There is a lot of activity in the calculation of TMDs and GPDs in various non-perturbative models, such as the Dyson-Schwinger approach, holography and AdS/QCD [15. In the Hamiltonian approach these distributions are related to eigen-solutions of the QCD light-front Hamiltonian. The distributions of the nucleon which can be measured at an EIC can be constructed from operators in lattice QCD calculations. GPDs can be constructed from local operators and lattice QCD calculations are more straightforward than for TMDs. In order to relate TMDs and other distributions to lattice QCD calculations there have been a number of novel techniques developed in recent years [16 18. Comparison of precise extractions of these distributions from experimental data with ab initio 


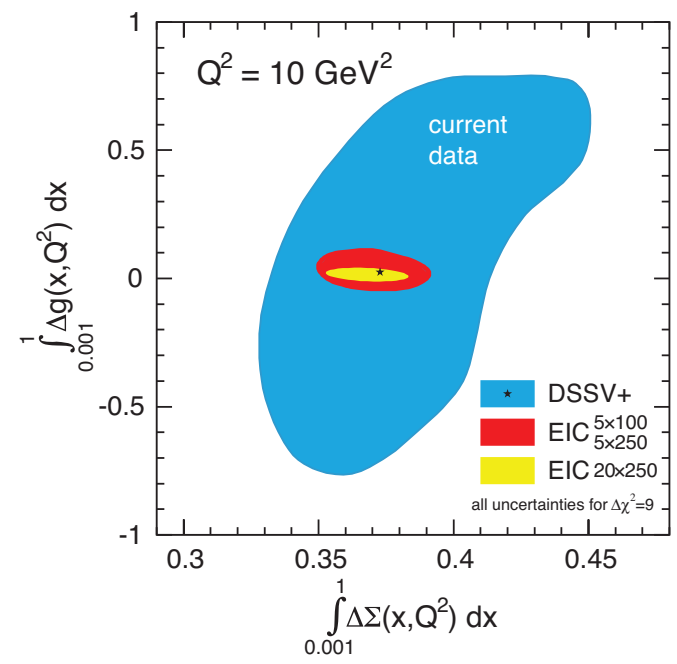

FIG. 3. (Color online) Current knowledge of contribution from quarks $\Delta \Sigma$ and gluons $\Delta G$ coming from the region of $0.001<x<1$ to the spin of the nucleon compared with projected uncertainty at an EIC at two different energies. Figure from Ref. [1].

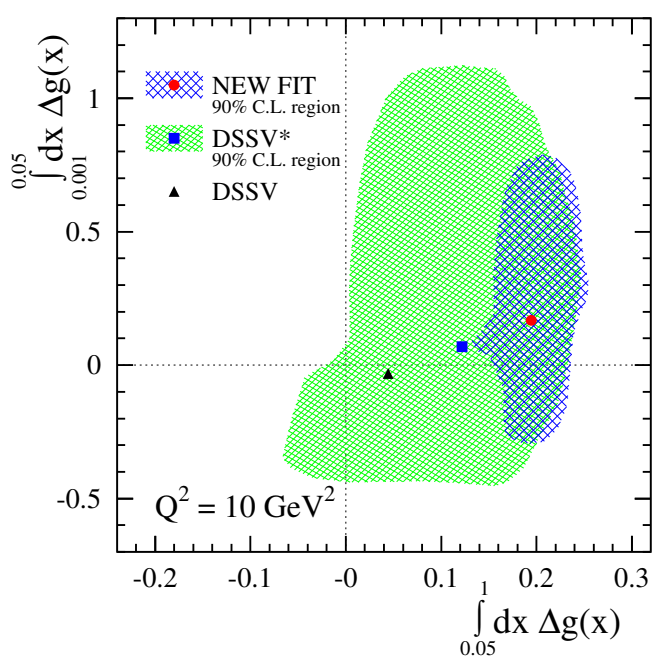

FIG. 4. (Color online) 90\% C.L. areas in the plane spanned by the truncated moments of $\Delta g$ computed for $0.05 \leq x \leq$ 1 and $0.001 \leq x \leq 0.05$ at $Q^{2}=10 \mathrm{GeV}^{2}$. Results for DSSV, DSSV ${ }^{*}$ and our new analysis, with the symbols corresponding the respective values of each central fit, are shown. Figure from Ref. [26]

calculations will be very important in the future. However, we emphasize, separation of the GPD helicity structures $(H, E, \tilde{H}, \tilde{E} \ldots)$ and different TMDs $\left(f_{1 T}^{\perp}, h_{1} \ldots\right)$ is feasible only with a facility capable of having both longitudinally and transversely polarized beams.

One of the key applications of GPDs is the "Ji-relation" 19 that allows one to relate the quark angular momentum (spin plus orbital) to the $x$-moment of GPDs. By subtraction, this also allows a determination of the quark orbital angular momentum as well as the gluon angular momentum. Significant progress has been made since the EIC White Paper to better interpret the terms that appear in the nucleon spin decomposition [20, 21] in terms of both twist-three quark-gluon correlations [22, 23, with also a natural description of orbital angular momentum in terms of Wigner functions [24, 25]. An EIC will be crucial to drive both the theoretical and experimental programs to understand and explore the partonic content of the nucleon spin.

Let us emphasize the requirements for EIC: A study of sea quark and gluon distributions for GPD and TMD programs is possible with a complete measurement of DVCS and deeply virtual meson production (DVMP) (including non diffractive pseudo scalar production) for GPDs and SIDIS measurements with different produced hadrons $\left(\pi^{ \pm}, K^{ \pm}, p, \bar{p}, \ldots\right)$ for TMDs and spectator proton tagging on polarized light-ion beams in both cases.

Both TMDs and GPDs are extremely important for our understanding of the nucleon as a dynamical relativistic system of quarks and gluons and represent future "text book knowledge", see Fig. 2. These distributions are related to the orbital motion of partons inside of the nucleon and ultimately will lead to better understanding of underlying dynamics of strong color force.

\section{Helicity structure of the nucleon:}

The past few decades have witnessed some extraordinary progress in the measurement and understanding of the helicity structure of the nucleon. The inclusive structure function $g_{1}\left(x, Q^{2}\right)$, whose unexpectedly small size for $x<0.1$ revived interest in the internal spin structure of the nucleon and gave rise to the "nucleon spin puzzle", is now measured down to $x \simeq 0.004$ for $Q^{2}>1 \mathrm{GeV}^{2}$. The RHIC spin physics program has yielded precision data on the beam-helicity dependent production rates for inclusive jets and pions in polarized proton-proton collisions, which in global QCD analyses give evidence for a positive polarization of gluons in the proton for $x \geq 0.05$. Further advances will come from ongoing analyses of RHIC data at the top energy of $\sqrt{s}=510 \mathrm{GeV}$, both for the polarization of gluons through jet measurements and the polarization of the light (anti)quarks through measurements of the spin-dependent rates of leptonic decays of weak bosons produced at this energy. They are also anticipated from proposed future running periods and from data with new instrumentation closer to the beam regions. In the large- $x$ region, the $12 \mathrm{GeV}$ program 
at Jefferson Lab is expected to determine the valence quark helicity distributions, settling decades-old questions about the behavior of polarized to unpolarized PDF ratios in the $x \rightarrow 1$ limit.

Despite these advances, it is currently not possible to conclusively answer the basic questions on the nucleon spin composition. The total contribution from gluon spin to the nucleon spin, for example, is uncertain to a level where it could be zero, or even larger than the nucleon spin itself, see Fig 3, which would preclude any conclusions on possible contribution from orbital angular momentum via the spin sum rule. The quark and anti-quark spin contributions are also currently limited by uncertainties from the unmeasured region at small $x$. However a recent global analysis [26] of new experimental data on the double longitudinal spin asymmetry in $p p$ scattering in jet and $\pi^{0}$ production at RHIC [27] finds a non trivial positive gluon polarization at intermediate momentum scales. These new results and analyses could indicate that gluons make a significant contribution to the nucleon spin sum rule. The integrated from gluon helicity $\Delta G\left(Q^{2}\right)$, is displayed in Fig 4 at $Q^{2}=10 \mathrm{GeV}^{2}$, and the contribution to $\Delta G\left(Q^{2}\right)$ from the region covered by experimental data indicate positive gluon polarization and there is a big uncertainty coming from the region of small $x$ which will be covered by EIC.

The proposed program with a polarized EIC brings unique capabilities to the study of the nucleon spin. In particular, an EIC will enable measurements that extend the kinematic range of existing and forthcoming data to smaller values of $x$ by between one and two orders of magnitude at the same resolution scales. At fixed values of $x$, the EIC will allow measurements over an unprecedented range in $Q^{2}$. The EIC White Paper [1] contains the impact from simulated measurements of $g_{1}\left(x, Q^{2}\right)$ on the (anti)quark and gluon spin contributions to the nucleon spin. Besides probing deeply into the gluon-dominated region of the nucleon, these measurements should lead to textbook knowledge on the fundamental composition of the nucleon spin. Semi-inclusive measurements with one or more identified hadrons in the final state will result in comparably impressive advances in delineating the flavor structure of the quark and anti-quark spin contribution [28. Furthermore, inclusive electro-weak measurements, utilizing both neutral and charged currents [29] will give access to novel structure functions and provide a unique avenue for probing the flavor decomposition of the polarized nucleon sea [30].

\section{Conclusion:}

In conclusion, an EIC will be a precision QCD collider probing nuclei in the region dominated by "sea" quarks and gluons. Its development represents an exciting opportunity to open the next QCD frontier for nuclear physics. It promises to answer fundamental outstanding questions about the structure of hadrons, such as the decomposition of the nucleon spin, as well provide an opportunity to unravel the three-dimensional sea quark and gluon structure of visible matter.

Acknowledgments: We thank all participants of the workshop and our colleagues for stimulating discussions. This work was supported in part by DOE contracts No. DE-AC05-06OR23177 (J.C.,W.M.,A.P.,D.R.,C.W.), DE-AC0298CH10886 (E.A., L.B., J.Q.), DE-AC02-05CH11231 (E.S., F.Y.), DE-AC02-76SF00515 (S.B.), DE-FG02-07ER41460 (L.G.), and DE-AC52-06NA25396 (Z.K.).

[1] A. Accardi, J. Albacete, M. Anselmino, N. Armesto, E. Aschenauer, et al., (2012), arXiv:1212.1701 [nucl-ex]

[2] Report to the Nuclear Science Advisory Committee (NSAC) on the Long Range Plan for Nuclear Physics 2007, Subcommittee Report on Performance Measures, 2008, 136 pp.

[3] D. Boer, M. Diehl, R. Milner, R. Venugopalan, W. Vogelsang, et al., (2011), arXiv:1108.1713 [nucl-th]

[4] M. Anselmino, H. Avakian, D. Boer, F. Bradamante, M. Burkardt, et al., Eur.Phys.J. A47, 35 (2011), arXiv:1101.4199 [hep-ex]

[5] A. Accardi, V. Guzey, A. Prokudin, and C. Weiss, Eur.Phys.J. A48, 92 (2012), arXiv:1110.1031 [hep-ph]

[6] M. Burkardt, Phys. Rev. D62, 071503 (2000), hep-ph/0005108; Int.J.Mod.Phys. A18, 173 (2003), arXiv:hep-ph/0207047 $[$ hep-ph].

[7] X. Ji, Phys. Rev. Lett. 91, 062001 (2003), hep-ph/0304037

[8] I. Balitsky, A. Prokudin, and A. Radyushkin, Int.J.Mod.Phys.Conf.Ser. 04 (2011); A. Radyushkin and A. Prokudin, 20, pp.1 (2012); 25 (2014).

[9] F. Gautheron et al. (COMPASS Collaboration), (2010), CERN-SPSC-2010-014:CERN-SPSC-2010-014.

[10] C. Aidala, N. Ajitanand, Y. Akiba, Y. Akiba, R. Akimoto, et al., (2012), arXiv:1207.6378 [nucl-ex].

[11] C. STAR, (2014), https://drupal.star.bnl.gov/STAR/files/pp.pA_.LoI_.pp_.pA_.v7.pdf.

[12] C. Brown, D. Geesaman, P. Reimer, D. Christian, M. Diefenthaler, et al., (2014), letter of Intent for a Drell-Yan Experiment with a Polarized Proton Target, FERMILAB-PROPOSAL-1039.

[13] E.-C. Aschenauer, S. Fazio, K. Kumericki, and D. Mueller, JHEP 1309, 093 (2013), arXiv:1304.0077 [hep-ph].

[14] P. Jimenez-Delgado, T. Hobbs, J. Londergan, and W. Melnitchouk, (2014), arXiv:1408.1708 [hep-ph]

[15] G. F. de Teramond and S. J. Brodsky, Phys.Rev.Lett. 102, 081601 (2009), arXiv:0809.4899 [hep-ph]; S. J. Brodsky, G. F. de Teramond, H. G. Dosch, and J. Erlich, (2014), arXiv:1407.8131 [hep-ph] 
[16] B. Musch, P. Hagler, M. Engelhardt, J. Negele, and A. Schafer, Phys.Rev. D85, 094510 (2012), arXiv:1111.4249 [hep-lat]

[17] X. Ji, Phys.Rev.Lett. 110, 262002 (2013), arXiv:1305.1539 [hep-ph].

[18] H.-W. Lin, J.-W. Chen, S. D. Cohen, and X. Ji, (2014), arXiv:1402.1462 [hep-ph].

[19] X. Ji, Phys. Rev. Lett. 78, 610 (1997), hep-ph/9603249, X.-D. Ji, Phys. Rev. D58, 056003 (1998), arXiv:hep-ph/9710290

[20] M. Burkardt, Phys.Rev. D88, 114502 (2013), arXiv:0810.3589 [hep-ph].

[21] E. Leader and C. Lorce, Phys.Rept. (2013), arXiv:1309.4235 [hep-ph]

[22] Y. Hatta, Phys.Lett. B708, 186 (2012), arXiv:1111.3547 [hep-ph]

[23] X. Ji, X. Xiong, and F. Yuan, Phys.Rev. D88, 014041 (2013), arXiv:1207.5221 [hep-ph]

[24] C. Lorce and B. Pasquini, Phys.Rev. D84, 014015 (2011), arXiv:1106.0139 [hep-ph]|

[25] X. Ji, X. Xiong, and F. Yuan, Phys.Rev.Lett. 109, 152005 (2012), arXiv:1202.2843 [hep-ph].

[26] D. de Florian, R. Sassot, M. Stratmann, and W. Vogelsang, Phys.Rev.Lett. 113, 012001 (2014), arXiv:1404.4293 [hep-ph].

[27] A. Adare et al. (PHENIX Collaboration), Phys.Rev. D90, 012007 (2014), arXiv:1402.6296 [hep-ex] L. Adamczyk et al. (STAR Collaboration), (2014), arXiv:1405.5134 [hep-ex]

[28] E. C. Aschenauer, R. Sassot, and M. Stratmann, Phys.Rev. D86, 054020 (2012), arXiv:1206.6014 [hep-ph].

[29] A. Adare et al. (PHENIX Collaboration), Phys.Rev.Lett. 106, 062001 (2011), arXiv:1009.0505 [hep-ex] L. Adamczyk et al. (STAR Collaboration), Phys.Rev.Lett. 113, 072301 (2014), arXiv:1404.6880 [nucl-ex].

[30] E. C. Aschenauer, T. Burton, T. Martini, H. Spiesberger, and M. Stratmann, Phys.Rev. D88, 114025 (2013), arXiv:1309.5327 [hep-ph] 